\title{
Markov approximation for multilayered selection in satellite network
}

\author{
LI Yiqing $^{1}$, LI Yuqing ${ }^{1}$, GAN Xiaoying ${ }^{1}$, WANG Jingchao ${ }^{2}$, XU Youyun ${ }^{3}$, WANG Xinbing ${ }^{1}$
}

1. Shanghai Jiao Tong University, Shanghai 200240, China

2. Institute of China Electronic System Engineering Corporation, Beijing 100039, China

3. PLA University of Science and Technology, Nanjing 210007, China

\begin{abstract}
A satellite network system comprises three layers of satellites: LEO (Low Earth Orbit), MEO (Middle Earth Orbit) and GEO (Geostationary Orbit). In the system, users can choose a layer according to their demands, including QoS (Quality of Service), congestion, energy cost, etc. The utility that users gain will change when they access satellites in different layers. The mobility of satellites in the LEO and MEO leads to frequent handover among satellites in the same layer. These characteristics of multi-layered satellite networks make it possible for us to exploit the optimal distribution of users, which will maximize the utility of the entire satellite network. While the proposed problem is an NP-hard problem, we analyze the system based on the Markov chain and use the Markov approximation to approach the maximum utility. In addition, we use the count down and select algorithm to implement the process of Markov chain. The simulation results validate the convergence of the Markov approximation. In addition, the gap between the approximate value and optimal values decreases with an increase in $\beta$, which is a positive constant in Markov formulation, according to the simulation results.
\end{abstract}

Key words: satellite network, multi-layer, maximum utility, Markov chain, Markov approximation

\section{Introduction}

The satellite network plays an increasingly important role in modern radio network development. Facebook has proposed a plan named "Connecting the world from the sky," which will try to connect the world via satellite network ${ }^{[1]}$. A satellite network system includes three layers: LEO (Low Earth Orbit), MEO (Middle Earth Orbit) and GEO (Geostationary Orbit). Generally, these three layers differ from each other in terms of services supported, capacity, cost of accessing and cost of handover. Chen, et al analyzed the characteristics of satellite networks ${ }^{[2]}$. In Ref.[3], Truchly, et al investigated two-layer satellite systems, which exhibits the performance similar to that of multilayered satellite networks.

In the satellite network system, all the users want to be served at a low cost, which will result in the layers with lower costs becoming congested while the expensive layers remain underutilized. In addition,

Manuscript received Sept. 8, 2016; accepted Sept. 26, 2016

This work is supported by the National Natural Science Foundation of China (Nos.61325012, 61428205, 91438115, 61532012, 61671478, 61672342). 
unfair distribution of users in the network will render the system incapable of obtaining the optimal utility, which means users cannot be provided with high quality service while some layers of the system are still underutilized.

Therefore, it is necessary to exploit the optimal distribution of users, which will provide the whole system with the maximum utility. The utility of satellite networks can be improved by distributing users to multiple layers or satellites. The loadbalanced algorithm can help the system avoid congestion and minimize the queuing delay in the system. In Ref.[4], Wang, et al proposed the Tailored Load-Aware Routing for load balance in multilayered satellite network, in which the optimal portion of network load is diverted through the upper layer. Jiang, et al used Stackelberg game based analysis to apply the load-balancing scheme in a hybrid GEOLEO satellite network ${ }^{[5]}$, which maximizes the average signal-to-noise ratio of the mobile terminals that need handover and optimizes the overall utility of the relays in terms of payment. When the layer is congested, the user can handover to the other layers to obtain higher QoS (Quality of Service) at the expense of cost. $\mathrm{Wu}$, et al discussed real time handover management in the mobile satellite communication networks ${ }^{[6]}$. In Ref.[7], Ong, et al introduced a novel measurement-based RAT (Radio Access Technology) selection technique that provided a practical solution to acquire QoS information. The method removed unnecessary handovers caused by Bayesian estimation when selecting the most suitable RAT.

However, finding the optimal solution of a combinational problem is an NP-hard problem, which can only be solved by exhaustive research. To reduce the complexity of calculation, Chen, et $\mathrm{al}^{[8]}$ proposed and proved that Markov approximation can be used to solve a combinational problem. In Ref.[9], Chen, et al used the Markov chain model to simulate the process of handover of users between different RATs. In addition, they proposed an effective algorithm to implement the process. However, it considers the terrestrial networks whereas we adopt the Markov chain model in multi-layer satellite networks. Chen, et al consider a constant user reward for the utility function; nevertheless, we assume that the multi-layer selection for users affects user reward. In particular, the user reward decreases when the number of users accessing the same layer increases. In addition, the energy cost is considered in our model. Therefore, the utility function is different. The convergence process of Markov approximation is different as well.

In this work, we aim to optimize multi-layer selection in a distributed manner. The handover process between three different layers can be formulated as a Markov chain model. We assume that each user has a unique demand and corresponding cost in different layers. A utility maximization problem for layer selection is proposed. However, we can only use an exhaustive search to obtain the optimal situation because the problem is NP-hard. In order to simplify the calculation process, we use the Markov approximation to approximate the optimal value of the problem. In addition, we modify the CDS (Count Down and Select) algorithm proposed in Ref.[9] to implement the process of multi-layer selection. Practically, the CDS algorithm helps decrease the time wasted in waiting for the whole system to reach its maximized utilities. Specifically, users decide whether to change their layer selections after knowing the utility values for the current layer. When all the users do not intend to change their layer selections, the utility of the whole networks reaches its maximum.

The rest of this paper is organized as follows. Section 2 presents the system model. Section 3 shows the process of Markov approximation and describes the CDS algorithm. The simulation results are presented in Section 4. Section 5 concludes the paper. 


\section{System model}

In the multilayer system, there are three types of satellites. The users can access one of them and handover to another in a different layer at a certain time to seek a higher QoS and higher system-level utility. The system composition is shown in Fig.1.

We assume that there is no interference between the satellites in this system and a user can only access one satellite at a time. In this paper, we did not consider the duration of service.

Each layer can be denoted as $l_{i}, i \in\{1,2,3\}$, which represents LEO, MEO and GEO, respectively. We assume that there are $N$ users in the whole satellite network. In the system, a configuration $f$ includes $N$ items and can be denoted by $f=\left\{a_{1}, a_{2}, a_{3}, \cdots, a_{N}\right\}$, where $a_{i}, i=1,2,3, \cdots, N$ indicates the layer each user has accessed and $\forall a_{i} \in\left\{l_{1}, l_{2}, l_{3}\right\}$. The configuration $f$ reflects the layer each user stays in and we consider $f \in F$, where $F$ is the set of $f$. Let $N_{i}(f)$ denote the number of users in $l_{i}$ under the configuration $f$. Therefore, we have

$$
N=\sum_{i=1,2,3} N_{i}(f),
$$

Each user has a unique demand for service and we denote it numerically as $x_{j}, j=1,2,3, \cdots, N$. In addition, each layer has a threshold value denoted as $T_{i}, i \in\{1,2,3\}$, which represents the threshold value of LEO, MEO and GEO, respectively. When a user's demand is under the threshold of the layer he is accessing, he will be served without penalty. In the system, the demands of users can be sorted into three types as follows.

$$
\left\{\begin{array}{l}
0 \leqslant x_{j} \leqslant T_{1}, \text { Lower demand users, } \\
T_{1}<x_{j} \leqslant T_{2}, \text { Moderate demand users, } \\
T_{2}<x_{j} \leqslant T_{3}, \text { Higher demand users. }
\end{array}\right.
$$

We call the lower demand users $S_{1}$ to make it easier to describe them. Similarly, moderate and higher demand users are called $S_{2}$ and $S_{3}$, respectively. However, all kinds of users can choose a satellite. If the user chooses the proper layer according to the above range (e.g., an $S_{1}$ chooses a satellite in the LEO layer), the QoS of the user will be influenced by the congestion and capacity of the satellites. in such a situation, we denote the award users gain as

$$
W_{\text {coca }}=\left(1-p_{i}\left(N_{i}(f)-1\right)\right) * \operatorname{sum}_{i j}(f),
$$

where $i, j \in\{1,2,3\}$ and $i \geqslant j$. We denote $p_{i}$ as the satellite capacity's influence factor, which indicates the impacts of the users' sensitivity to satellite congestion on their gain. The more sensitive the

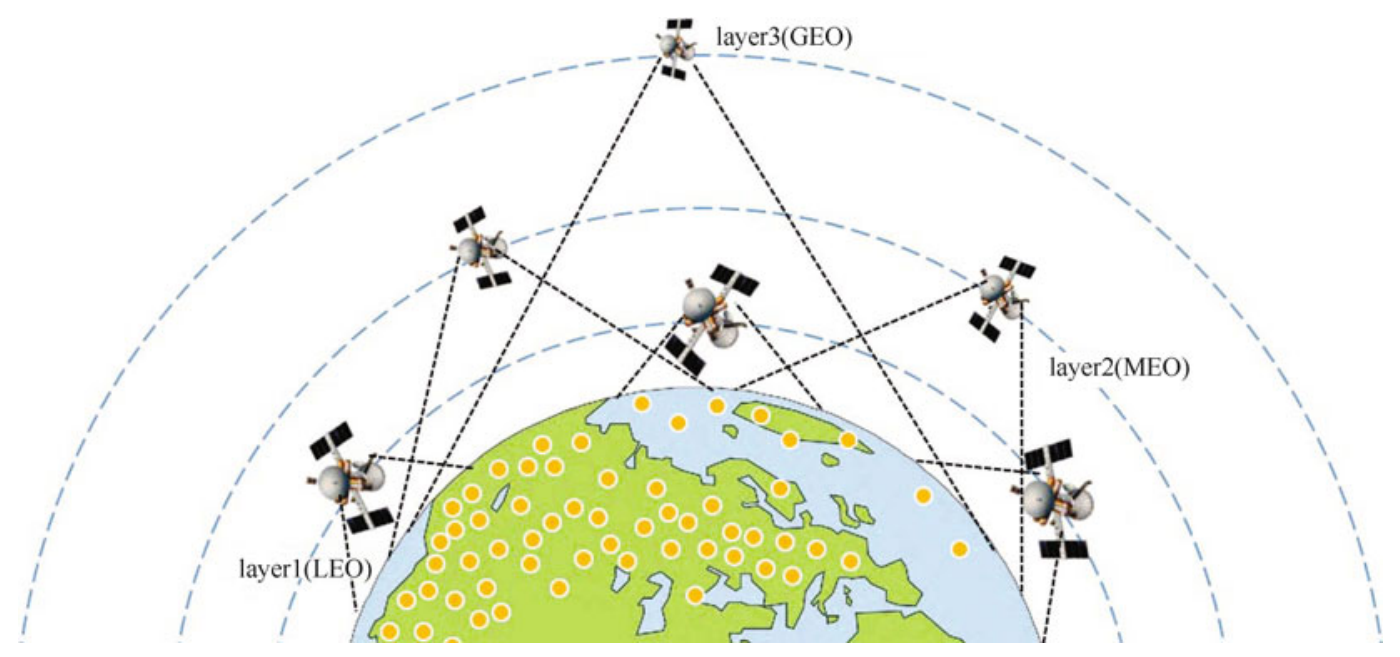

Figure 1 Example of multi-layer satellite network 
users are to networks, the higher the value of $p_{i}$. Specifically, for satellites in the GEO layer with large capacities, their users will be less affected by the congestion. Therefore, $p_{i}$ is small in this case. The $\operatorname{sum}_{i j}(f), i, j \in\{1,2,3\}$ represents the sum of the $S_{j}$ s' demands in layer $i$, under configuration $f$. The term 1$p_{i}\left(N_{i}(f)-1\right)$ represents the users gain per demand based on the population. Therefore, we can obtain the $W_{\text {coca }}$ by multiplying $\operatorname{sum}_{i j}(f)$ and $1-p_{i}\left(N_{i}(f)-1\right)$. In this equation, $i, j$ meet the condition $i \geqslant j$, which means the demands of $S_{j}$ s are under the threshold of layer $i$. When the users' demand exceeds the threshold value of the layer that the user is accessing, the QoS of the user will be influenced by the capacity and the congestion. It can be denoted as $q_{i j}, i, j \in\{1,2$, 3 , which represents the influence on an $S_{j}$ when he is accessing a satellite present in layer $i$. Under this situation, we denote the award users gain as

$$
W_{\text {other }}=q_{i j}\left(1-p_{i}\left(N_{i}(f)-1\right)\right) * \operatorname{sum}_{i j}(f),
$$

where $i, j \in\{1,2,3\}$ and $i<j$. In this equation, $i$ and $j$ meet the condition $i<j$, which means the demands of $S_{j} \mathrm{~s}$ are above the threshold of layer $i$.

The satellite system is operating outside the Earth. Users must pay a higher amount in order to access satellites compared with that required for accessing terrestrial networks. In the system, the cost of accessing is denoted as $m_{i}, i \in\{1,2,3\}$. In addition, according to the distance of each layer from the Earth, these three related coefficients should meet $m_{1}<m_{2}$ $<m_{3}$. The total cost of accessing layer $i$ for $S_{j} s$ under configuration $f$ is denoted as

$$
M_{i j}=m_{i} \operatorname{sum}_{i j}(f), i, j \in\{1,2,3\} .
$$

The difference between the satellite network and terrestrial network is that users need to handover between the satellites in the same layer when they have service in the satellite layers. Considering that the satellite in the GEO layer is geostationary, there is no need for users to handover in this layer. However, when a user access an LEO or MEO layer, the handover in the same layer generates additional costs because the velocity of orbiting is greater than the rotational velocity of the Earth. In the system, the cost of handover in the same layer is denoted as $k_{i}, i \in\{1,2$, $3\}$. According to the relative velocity of the Earth, these three coefficients should meet $k_{1}>k_{2}>k_{3}$, in which $k_{3}=$ 0 . The total cost of handover of $S_{j} \mathrm{~s}$ in layer $i$ under the configuration $f$ is denoted as $K_{i j}$.

$$
K_{i j}=k_{i} * \operatorname{sum}_{i j}(f), i, j \in\{1,2,3\} .
$$

The utility of each layer under the configuration $f$ is denoted as $U_{i}(f), i \in\{1,2,3\}$. According to Eqs. (3), (4), (5) and (6), the utility function of each layer is as follows.

$$
U_{1}(f)=\left\{\begin{array}{l}
\left(1-p_{1}\left(N_{1}(f)-1\right)\right) \operatorname{sum}_{11}(f) \\
-m_{1} \operatorname{sum}_{11}(f)-k_{1} \operatorname{sum}_{11}(f), \\
q_{12}\left(1-p_{1}\left(N_{1}(f)-1\right)\right) \operatorname{sum}_{12}(f) \\
-m_{1} \operatorname{sum}_{12}(f)-k_{1} \operatorname{sum}_{12}(f), \\
q_{13}\left(1-p_{1}\left(N_{1}(f)-1\right)\right) \operatorname{sum}_{13}(f) \\
-m_{1} \operatorname{sum}_{13}(f)-k_{1} \operatorname{sum}_{13}(f) .
\end{array}\right.
$$

$$
U_{2}(f)=\left\{\begin{array}{l}
\left(1-p_{2}\left(N_{2}(f)-1\right)\right) \operatorname{sum}_{21}(f) \\
-m_{2} \operatorname{sum}_{21}(f)-k_{2} \operatorname{sum}_{21}(f), \\
\left(1-p_{2}\left(N_{2}(f)-1\right)\right) \operatorname{sum}_{22}(f) \\
-m_{2} \operatorname{sum}_{22}(f)-k_{2} \operatorname{sum}_{22}(f), \\
q_{23}\left(1-p_{2}\left(N_{2}(f)-1\right)\right) \operatorname{sum}_{23}(f) \\
-m_{2} \operatorname{sum}_{23}(f)-k_{2} \operatorname{sum}_{23}(f) .
\end{array}\right.
$$

$$
U_{3}(f)=\left\{\begin{array}{l}
\left(1-p_{3}\left(N_{3}(f)-1\right)\right) \operatorname{sum}_{31}(f) \\
-m_{3} \operatorname{sum}_{31}(f)-k_{3} \operatorname{sum}_{31}(f), \\
\left(1-p_{3}\left(N_{3}(f)-1\right)\right) \operatorname{sum}_{32}(f) \\
-m_{3} \operatorname{sum}_{32}(f)-k_{3} \operatorname{sum}_{32}(f), \\
\left(1-p_{3}\left(N_{3}(f)-1\right)\right) \operatorname{sum}_{33}(f) \\
-m_{3} \operatorname{sum}_{33}(f)-k_{3} \operatorname{sum}_{33}(f) .
\end{array}\right.
$$

Our object function is to maximize the utility of the whole satellite system by choosing the optimum distribution of users. The utility maximization problem can be expressed as follows.

$$
\max _{f \in F} \sum_{i=1,2,3} N_{i}(f) .
$$




\section{Markov approximation and implement algorithm}

The utility maximization problem in a satellite net work system is NP-hard and can only be solved through exhaustive search. In this section, we will describe the Markov approximation and CDS algorithm proposed in Ref.[9].

\subsection{Markov approximation}

The equivalent formulation of the utility maximization problem can be written as

$$
\begin{aligned}
& \max _{p \geqslant 0} \sum_{f \in F} p_{f} \sum_{i=1,2,3} U_{i}(f), \\
& \text { s.t. } \sum_{f \in F} p_{f}=1
\end{aligned}
$$

where $p_{f}$ is the percentage of the time during which the system stays in the configuration $f$. We regard $\sum_{i=1,2,3} U_{i}(f)$ as the weight of $f$, and we need to find the maximum weighted configuration for the Markov chain. The problem in this paper is a combinational problem. The size of set $F$ is very large even for a small number of users, which makes the problem hard to calculate. We can only use exhaustive search to get the accurate result of the problem, which produces high calculation complexity. Therefore, Markov approximation is a better way to solve the problem approximately. For a positive constant $\beta$ and $n$ non-negative real variables $y_{1}, y_{2}, \cdots, y_{n}$, we have

$$
\begin{aligned}
\max \left(y_{1}, \cdots, y_{n}\right) & \leqslant \frac{1}{\beta} \log \left(\exp \left(\beta y_{1}\right)\right)+\cdots+\exp \left(\beta y_{n}\right) \\
& \leqslant \max \left(y_{1}, \cdots, y_{n}\right)+\frac{1}{\beta} \log n
\end{aligned}
$$

When $\beta$ approaches infinity, we have the formula

$\max \left(y_{1}, \cdots, y_{n}\right)=\lim _{\beta \rightarrow \infty} \frac{1}{\beta} \log \left(\exp \left(\beta y_{1}\right)+\cdots+\exp \left(\beta y_{n}\right)\right)$.

$$
y_{1}=\sum_{i=1,2,3} U_{i}\left(f_{j}\right), f_{j} \in F, j=1, \cdots, n .
$$

Thus, we have

$$
\max _{f \in F} \sum_{i=1,2,3} U_{i}(f) \approx \frac{1}{\beta} \log \left(\sum_{f \in F} \exp \left(\beta \sum_{i=1,2,3} U_{i}(f)\right)\right),
$$

where $\beta$ is a positive constant. It is known as the logsum-exp approximation, and its accuracy depends on the value of $\beta$. According to the log-sum-exp approximation, we have an approximate version of Eq.(15). It can be expressed as

$$
\begin{aligned}
& \max _{p \geqslant 0} \sum_{f \in F} p_{f} \sum_{i=1,2,3} U_{i}(f)-\frac{1}{\beta} \sum_{f \in F} p_{f} \log p_{f}, \\
& \text { s.t. } \sum_{f \in F} p_{f}=1 .
\end{aligned}
$$

From Refs.[8] and [9], we know that the error upper bound of the approximation will decrease compared with the absolute result as $\beta$ is increased. That means when $\beta$ increases, the results of Markov approximation will become increasingly accurate.

We have mentioned $p_{f}$ at the beginning of this section. Here, we define it as

$$
p_{f}=\frac{\exp \left(\beta \sum_{i=1,2,3} U_{i}(f)\right)}{\sum_{f^{\prime} \in F} \exp \left(\beta \sum_{i=1,2,3} U_{i}\left(f^{\prime}\right)\right)}, \forall f \in F .
$$

Therefore, the object function can be expressed as

$$
\max \sum_{f \in F} p_{f} \sum_{i=1,2,3} U_{i}(f) .
$$

\subsection{The CDS algorithm}

The CDS algorithm proposed in Ref.[9] is a distributed algorithm that can solve the problem in this paper. A new coefficient $p_{f f}$ is proposed, which represents the probability that users stay in the new layer once they handover.

$$
p_{f f^{\prime}}=\frac{\exp \left(\beta \sum_{i=1,2,3} U_{i}\left(f^{\prime}\right)\right.}{\max \left\{\exp \left(\beta \sum_{i=1,2,3} U_{i}\left(f^{\prime}\right)\right), \exp \left(\beta \sum_{i=1,2,3} U_{i}(f)\right)\right\}} .
$$


Initially, each user randomly enters a layer. Each layer operator will calculate the utility of the layer. Each user generates an exponentially distributed random number with a mean equals of $\gamma$. The user counts down according to the number. When the number of the user expires, he/ she will randomly handover to the other layer, which will lead the system to a new configuration $f^{\prime}$. The probability of user staying in the new layer is $p_{f f^{\prime}}$, whereas that of moving back to the original layer is $1-p_{f f^{\prime}}$. As shown in Eq.(19), when $\exp \left(\beta \sum_{i=1,2,3} U_{i}\left(f^{\prime}\right)\right) \geqslant \exp \left(\beta \sum_{i=1,2,3} U_{i}(f)\right)$, (i.e., the new RAT offers better performance), user $r$ will stay in the new layer with probability 1 . When $\exp \left(\beta \sum_{i=1,2,3} U_{i}\left(f^{\prime}\right)\right)<\exp \left(\beta \sum_{i=1,2,3} U_{i}(f)\right)$ (i.e., the original layer offers the better performance), the system will change back to the original configuration with a probability of $1-p_{f f}$ '. The process of the CDS algorithm is presented in Algorithm 1.

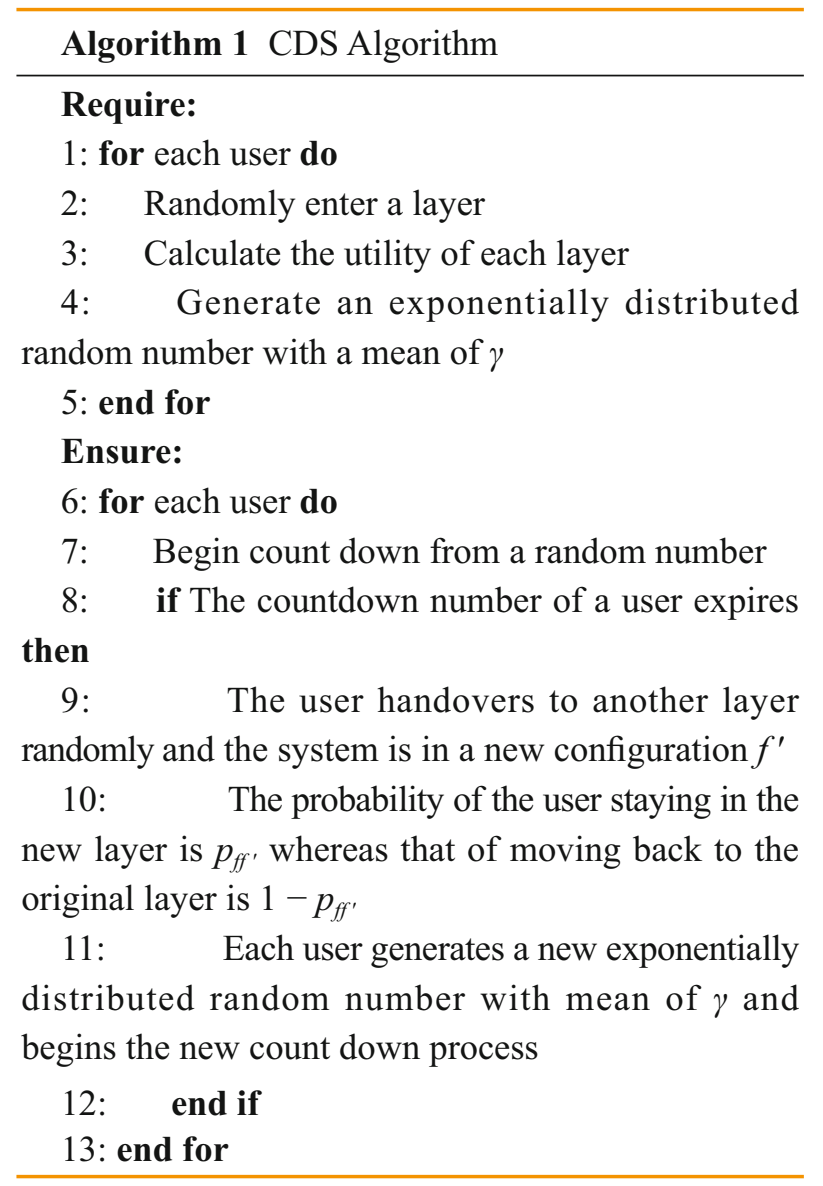

\section{Simulation}

In this section, numerical results are presented to validate the effectiveness of the model using the CDS algorithm. We assume there are 50 users in the entire satellite multilayer system. At the beginning, we generate 50 random numbers in the range $(0,1)$, which represent the demands of different users. The utility of the system depends on the distribution of users and the coefficients mentioned in section 2. In Tab.1, we give an example of the coefficients. In addition, we let $q_{12}=0.625, q_{13}=0.5$ and $q_{23}=0.8$.

Table 1 Coefficients of the function of system model

\begin{tabular}{cccc}
\hline \multirow{2}{*}{ impact factor } & \multicolumn{3}{c}{ layer } \\
\cline { 2 - 4 } & LEO & MEO & GEO \\
\hline capacity influence $\left(p_{i}\right)$ & 0.001 & 0.0001 & 0.00000001 \\
accessing cost $\left(m_{i}\right)$ & 0.1 & 0.15 & 0.2 \\
handover cost $\left(k_{i}\right)$ & 0.1 & 0.05 & 0 \\
\hline
\end{tabular}

Fig.2 illustrates the process of Markov approximation of the problem. We consider the impacts of $\beta$ and $\gamma$ on the utility. In Fig.2(a), we set the value of $\beta$ equal to 1, 10 and 30. From Ref.[9] and Fig.2(a), it is obvious that as the value of $\beta$ increases, the gap between the approximate and absolute values decreases. In addition, it illustrates that the convergence time decreases when the value of $\beta$ increases. In Fig.2(b), $\gamma$ is set as 1, 5 and 10. We can see that as the value of $\gamma$ increases, the gap between the approximate value and absolute value decreases.

Fig. 3 illustrates the dynamics of the number of users in each layer in the process of Markov approximation compared with results obtained through exhaustive search with $\beta=30$ and $\gamma=10$. Fig.3(a), Fig.3(b) and Fig.3(c) show the results for the LEO, MEO and GEO layers respectively. According to the model built at the beginning, we know each 
(a)

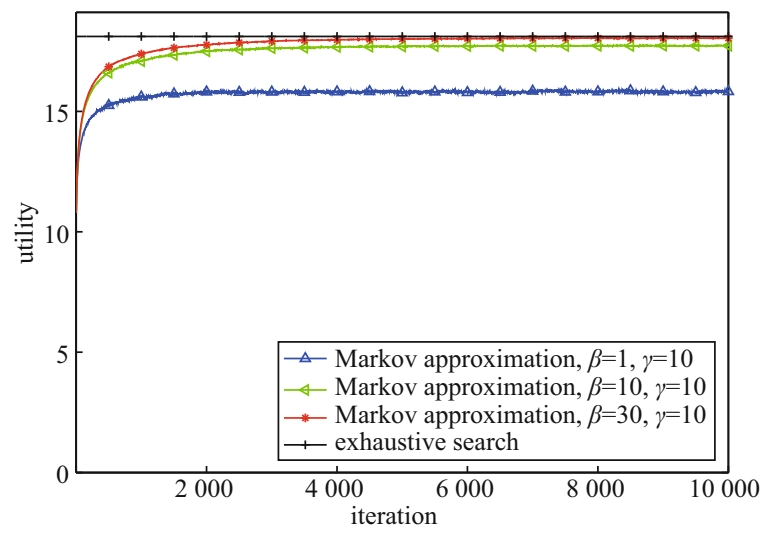

(b)

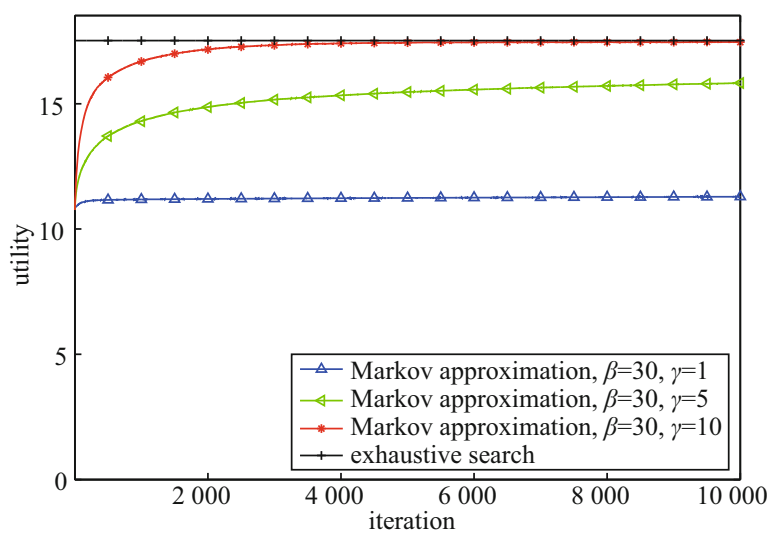

Figure 2 Process and result of Markov approximation: (a) different $\beta$; (b) different $\gamma$

(a)

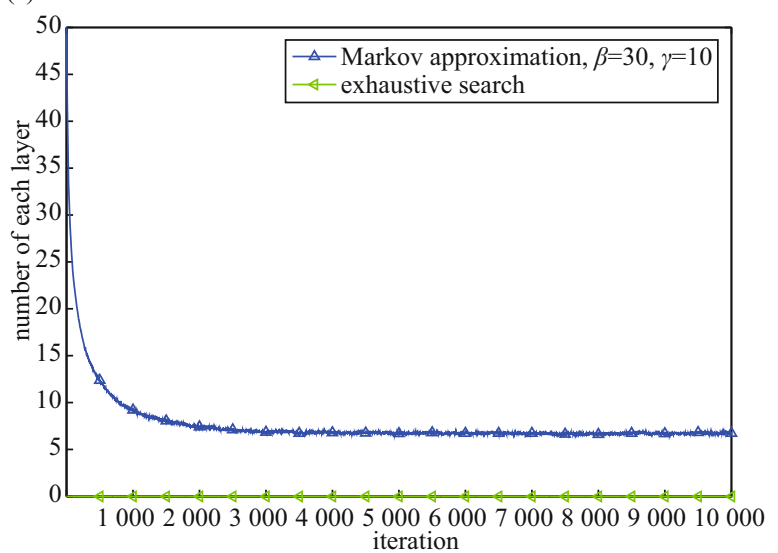

(b)

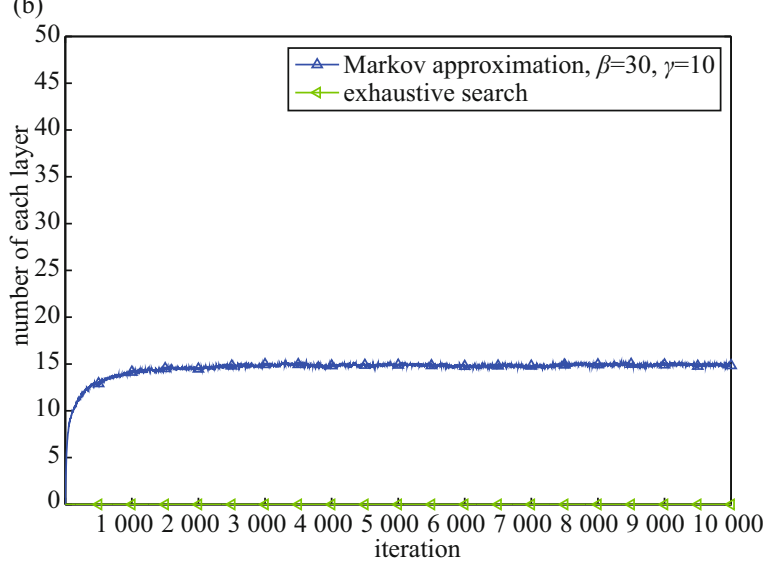

(c)

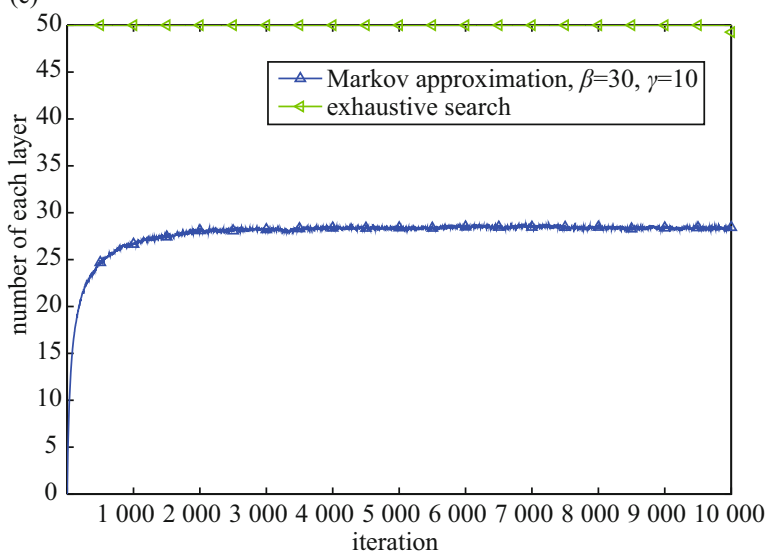

Figure 3 Dynamics of the number of users in the process of Markov approximation: (a) LEO; (b) MEO; (c) GEO

user will choose a proper layer to be served with higher QoS and lower cost. Users will keep adjusting the layer they access, and the layer operator will calculate the utility of users in each layer. In the process of Markov approximation, when the total utility of whole satellite system reaches the maximum value point, the distribution of users will incline to a stable status. 


\section{Conclusion}

This work used Markov chain to analyze the multilayer satellite network system. We build the mathematical model according to the characteristics of each layer. Finding the optimal distribution and maximum utility is an NP-hard problem, which can be solved approximately using Markov approximation. In order to implement the handover process, we modified the CDS algorithm proposed in Ref.[9] to implement the process of our model. The simulation results show that Markov approximation is an effective way to obtain the maximum utility unlike the exhaustive search. Markov approximation simplifies the complexity of calculation and reduces the time required to obtain the result. In addition, with the increase of $\beta$, the approximate value of total utility will get closer to the optimal value and the convergence time will decrease.

\section{References}

[1] Connecting the world from the sky[EB/OL]. http://fbcdndragon-a.akamaihd.net/hphotos-ak-ash3/t39.2365-6/851574
6115447522655401262758947 n.pdf.

[2] CHEN Z K, WEI X. Analysis of Satellite Communication Network Characteristics[C]//Communication Systems and Network Technologies (CSNT), Bhopal, India, 2014: 317-320.

[3] PETER T, MATE V. Performance of multilayered satellite networks $[\mathrm{C}] / /$ The 54th International Symposium ELMAR, Zadar, Yugoslavia, 2012: 113-116.

[4] WANG Y, SHENG M, LUI K S, et al. Tailored Load-Aware Routing for Load Balance in Multilayered Satellite Networks[C]// IEEE 82nd Vehicular Technology Conference (VTC Fall), Boston, USA, 2015:1-5.

[5] JIANG L Y, CUI G F, LIU S J, et al. Cooperative relay assisted load balancing scheme based on stackelberg game for hybrid GEO-LEO satellite network[C]//International Conference on Wireless Communications \& Signal Processing (WCSP), Nanjing, China, 2015: 1-5.

[6] WU Z F, HU G Y, YOUNES S, et al. A simple real-time handover management in the mobile satellite communication networks[C]// The 17th Asia-Pacific Network Operations and Management Symposium (APNOMS), Busan, Korea, 2015: 175-179.

[7] ONG E H, KHAN J Y. On optimal network selection in a dynamic multi-rat environment[J]. IEEE Communications letters, 2010, 14, (3): 217-219.

[8] CHEN M H, LIEW S C, SHAO Z Y. Markov approximation for combinatorial network optimization[J]. IEEE transactions on information theory, 2013, 59(10): 6301-6327.

[9] CHEN S W, GAN X Y, FENG X X, et al. Markov approximation for multi-RAT selection[C]//IEEE International Conference on Communications (ICC), London, UK, 2015: 3045-3050.

\section{About the authors}

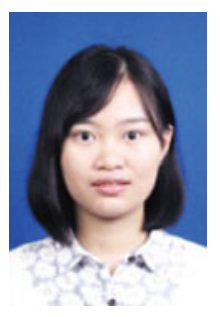

LI Yiqing received B.E. degree in information engineering from Shanghai Jiao Tong University, China. She is now pursuing the master degree in electronic engineering at Shanghai Jiao Tong University, Shanghai, China. Her research interests include network economics, social networks and satellite information networks.

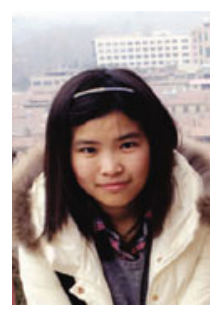

LI Yuqing received the B.S. degree in electronic engineering from Xidian University, Xi'an, China, in 2014, and is currently pursuing the Ph.D. degree in electronic engineering at Shanghai Jiao Tong University, Shanghai, China. Her current research interests include network economics, social aware networks, heterogeneous cellular networks, and network security.

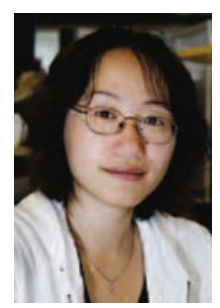

GAN Xiaoying [corresponding author] received the $\mathrm{Ph} . \mathrm{D}$. degree in electronic engineering from Shanghai Jiao Tong University, Shanghai, China, in 2006. She is currently with the Institute of Wireless Communication Technology, in the Department of Electronic Engineering, SJTU (Shanghai Jiao Tong University), where she is an associate professor. From 2009 to 2010, she worked as a visiting researcher at California Institute for Telecommunications and Information Technology (Calit2), University of California San Diego, California. Her current research interests include network economics, social aware networks, heterogeneous cellular networks, cognitive networks, multi-user multi-channel access, and dynamic radio resource management. (Email: ganxiaoying@sjtu.edu.cn) 


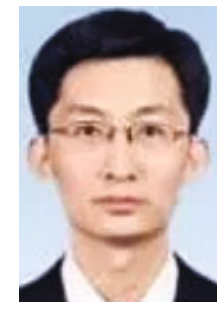

WANG Jingchao is a senior engineer in China Electronic Equipment System Engineering Company. His research interests include satellite information networks, top-level design and key technologies for satellite communication system system design.

XU Youyun was born in 1966, graduated from

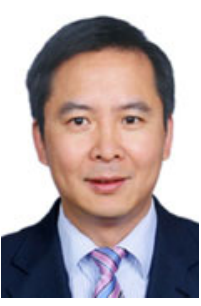
Shanghai Jiao Tong University with Ph.D. degree in information and communication engineering in 1999. He is currently a professor in Nanjing Institute of Communication Engineering, PLA University of Science and Technology, China. He is also a part-time professor with the Institute of Wireless Communication Technologies of SJTU (Shanghai Jiao Tong University), China. His research interests are focusing on new generation wireless mobile communication system (LTEIM-TAdvanced and related), advanced channel coding and modulation techniques, multiuser information theory and radio resource management, wireless sensor networks, cognitive radio networks, etc. He is a senior member of the IEEE and a senior member of Chinese Institute of ElectronicsIEICE member.

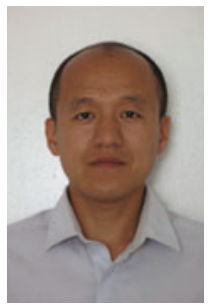

WANG Xinbing received the B.S. degree (with hons.) from the Department of Automation, Shanghai Jiao Tong University, Shanghai, China, in 1998, and the M.S. degree from the Department of Computer Science and Technology, Tsinghua University, Beijing, China, in 2001. He received the Ph.D. degree, major in the Department of Electrical and Computer Engineering, minor in the Department of Mathematics, North Carolina State University, Raleigh, in 2006. Currently, he is a professor in the Department of Electronic Engineering, Shanghai Jiao Tong University, Shanghai, China. Dr. Wang has been an associate editor for IEEE/ACM Transactions on Networking and IEEE Transactions on Mobile Computing, and the member of the Technical Program Committees of several conferences including ACM MobiCom 2012, ACM MobiHoc 2012, 2013, IEEE INFOCOM 2009-2014. 